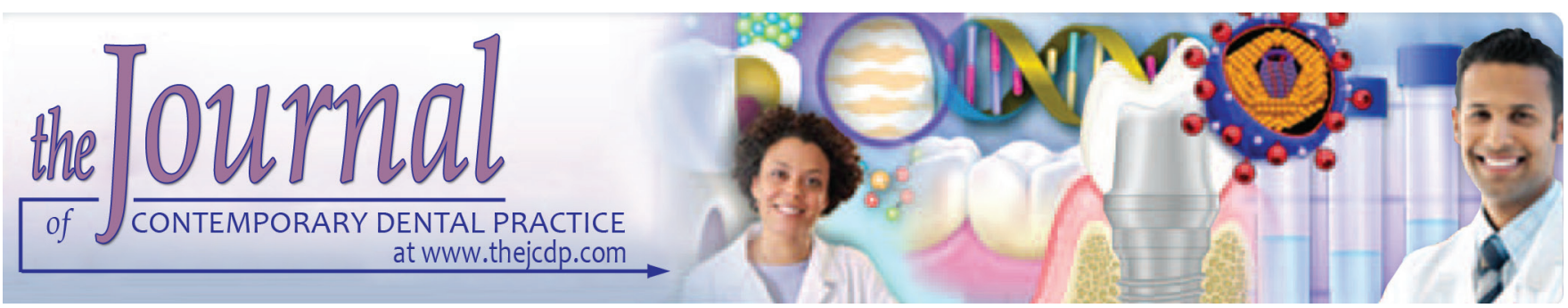

\title{
Influence of Different Etching Modes on Bond Strength to Enamel using Universal Adhesive Systems
}

\author{
${ }^{1}$ Ana CS Diniz, ${ }^{2}$ Matheus C Bandeca, ${ }^{3}$ Larissa M Pinheiro, ${ }^{4}$ Lauber J dos Santosh Almeida Jr, ${ }^{5}$ Carlos RG Torres \\ ${ }^{6}$ Alvaro H Borges, ${ }^{7}$ Shelon CS Pinto, ${ }^{8}$ Mateus R Tonetto, ${ }^{9}$ Rudys R De Jesus Tavarez, ${ }^{10}$ Leily M Firoozmand
}

\begin{abstract}
Introduction: The adhesive systems and the techniques currently used are designed to provide a more effective adhesion with reduction of the protocol application. The objective of this study was to evaluate the bond strength of universal adhesive systems on enamel in different etching modes (self-etch and total etch)
\end{abstract}

Materials and methods: The mesial and distal halves of 52 bovine incisors, healthy, freshly extracted, were used and divided into seven experimental groups $(n=13)$. The enamel was treated in accordance with the following experimental conditions: FUE-Universal System - Futurabond U (VOCO) with etching; FUWE - Futurabond U (VOCO) without etching; SB-Total Etch System - Single Bond 2 (3M); SBUE-Universal System - Single Bond Universal (3M ESPE) with etching; SBUWE - Single Bond Universal (3M ESPE) without etching; CLE-Self-etch System - Clearfil SE Bond (Kuraray) was applied with etching; CLWE - Clearfil SE Bond (Kuraray) without etching. The specimens were made using the composite spectrum TPH (Dentsply) and stored in distilled water $\left(37 \pm 1^{\circ} \mathrm{C}\right)$ for 1 month. The microshear test was performed using the universal testing machine EMIC DL 2000 with the crosshead speed of $0.5 \mathrm{~mm} / \mathrm{minute}$. The bond strength values were analyzed using statistical tests (Kruskal-Wallis test and Mann-Whitney test) with Bonferroni correction.

\footnotetext{
1,2,9 Department of Post-Graduate Program in Dentistry, CEUMA University, Renascença, São Luís, Brazil

${ }^{3,4,10}$ Department of Dentistry, Federal University of Maranhão (UFMA), São Luís, Brazil

${ }^{5}$ Department of Restorative Dentistry, Institute of Science and Technology of São José dos Campos - São Paulo State University - UNESP, São José dos Campos, Brazil

${ }^{6,8}$ Department of Post-Graduate Program in Integrated Dental Science, University of Cuiabá, Cuiabá, Brazil

${ }^{7}$ Department of Dentistry, State University of Ponta Grossa Ponta Grossa, Brazil

Corresponding Author: Ana CS Diniz, Department of PostGraduate Program in Dentistry, CEUMA University, Renascença São Luís, Brazil, e-mail: carol_diniz5@hotmail.com
}

Results: There was no statistically significant difference between groups $(p<0.05)$, where FUE $(36.83 \pm 4.9 \mathrm{MPa})$ showed the highest bond strength values and SBUWE $(18.40 \pm 2.2 \mathrm{MPa})$ showed the lowest bond strength values. The analysis of adhesive interface revealed that most failures occurred between the interface composite resin and adhesive.

Conclusion: The universal adhesive system used in dental enamel varies according to the trademark, and the previous enamel etching for universal systems and the self-etch both induced greater bond strength values.

Clinical significance: Selective enamel etching prior to the application of a universal adhesive system is a relevant strategy for better performance bonding.

Keywords: Acid etching, Adhesive systems, Shear strength.

How to cite this article: Diniz ACS, Bandeca MC, Pinheiro LM, dos Santos Almeida LJr, Torres CRG, Borges AH, Pinto SCS, Tonetto MR, De Jesus Tavarez RR, Firoozmand LM. Influence of Different Etching Modes on Bond Strength to Enamel using Universal Adhesive Systems. J Contemp Dent Pract 2016;17(10):820-825.

Source of support: The authors are grateful to the assistance provided by the Foundation of Research and Scientific and Technological Development of Maranhão (FAPEMA) and Institutional Scholarship Program for Scientific Initiation PIBIC-CEUMA.

Conflict of interest: None

\section{INTRODUCTION}

The simplification of procedures performed daily in dental practice is a great gain for dentists. Thus, the adhesive systems and the techniques currently used are designed to provide a more effective adhesion with reduction of the protocol application. ${ }^{1}$ Thus, the introduction of new materials and changes in the chemical composition can lead to an improvement in the quality of adhesion.

Etch-and-rinse and the total-etch techniques are applied through the full conditioning of dental substrate 
with phosphoric acid followed by the application of primer and bond, which can be presented in a single or in separate bottles. $^{2}$ The self-etching systems are relevant because of the convenience, simplicity, and time saving by eliminating the need for acid etching before its application. ${ }^{3}$

When phosphoric acid is used, a pattern more remarkable and retentive is found in enamel. The use of total-etch adhesive systems is usually preferred for indirect restorations or when there is the presence of large areas of enamel. ${ }^{4}$ In dentin, it is observed that the use of $37 \%$ phosphoric acid, in some cases, may promote excessive demineralization by avoiding proper infiltration and impregnation of resin in the collagen fibers, ${ }^{5}$ compromising, therefore, the union, unlike what happens when self-etch adhesives are used. ${ }^{6}$ However, enamel etching seems to promote the best adhesion results for the self-etch adhesive systems. ${ }^{7}$

Considering the number of clinical steps, a more versatile adhesive system has been developed that can be used as etch-and-rinse (two steps) or self-etch (one or two steps) mode. These new materials are "multipurpose," "multimode," or "universal" adhesives. ${ }^{8}$ Currently, studies 9,10 are being conducted in order to verify the applicability and efficiency of these materials on dental substrates, and some results have shown that the universal systems seem to be material dependent. ${ }^{8}$ Some results seem to indicate that this new category of adhesives when used in the dentin, with or without etching, presents lower bond strength values, nanoleakage, and degree of conversion when compared with self-etch and the already established total-etch adhesive systems. ${ }^{8}$

However, the effectiveness of multipurpose or universal adhesives in dental enamel is a factor that deserves to be studied, once adhesive systems are tested in vitro and compared with the results and parameters previously established before being used in the patient. ${ }^{11}$

According to the questions raised as well as the need for a study that improves its use in daily clinical practice, the objective of this study was to evaluate the bond strength of different universal adhesive systems with or without enamel pretreatment with $37 \%$ phosphoric acid, comparing them with total-etch and self-etch systems. The null hypothesis in this study is that there is no statistically significant difference in bond strength with or without enamel etching and between the tested adhesive systems (etch-and-rinse, self-etching, and multipurpose systems).

\section{MATERIALS AND METHODS}

A total of 52 healthy, freshly extracted, bovine incisors were employed and stored in distilled water. The teeth were sectioned at the cervical third with carborundum disk, and the roots were discarted. This study was approved by the Ethics Committee on Animal Use CEUA UNICEUMA, number 09/2013.
The coronal pulp was removed by the aid of dentin curette (Duflex Lucas \#86, SS White, Rio de Janeiro, RJ, Brazil) and endodontic files of type Kerr, and pulp chamber was irrigated with distilled water and dried with brief jets of air. The pulp chamber was obliterated with wax (Epoxiglass Ind Com, Diadema, São Paulo, Brazil) to prevent the penetration of acrylic resin. The bovine teeth were divided into mesial and distal part, so each adhesive system applied with or without acid etching was applied to one of these parts of the same tooth. Seven experimental groups with 13 samples each were formed. The specimens were placed in polyvinyl chloride pipe for embedding in acrylic resin (Jet-classical, São Paulo, São Paulo, Brazil) in order to allow the correct positioning of dental crowns for making the samples with composite resin. These samples were attached to a walrus that allowed the positioning of the vestibular crowns parallel to the microshear device.

Adhesive systems, following the specifications indicated by the manufacturer (Table 1), were used. The groups were divided according to the type of adhesive treatment performed: FUE-System Universal-Futurabond U (VOCO) with acid etching; FUWE - Futurabond U (VOCO) without etching; SB-Total etch system - Single Bond 2 (3M/ESPE); SBUE-Universal System - Universal Single Bond (3M/ESPE) with acid etching; SBUWE Single Bond Universal (3M/ESPE) without etching; CLESelf etch system - Clearfil SE Bond (KURARAY) with acid etching; CLWE-Self etch system - Clearfil SE Bond (KURARAY) without etching.

The specimens of the FUE, SBUE, and CLE groups were prepared at the mesial part of the teeth and those of FUWE, SBUWE, and CLWE groups were made in the distal part. The SB group was a control group, where the specimens were randomly manufactured using the mesial and distal halves. The buccal surfaces of the teeth were smoothed with grit paper 220 for 5 seconds, and then prophylaxis was performed with a mixture of pumice and water, applied with Robson brush for 10 seconds; for every 10 applications the brush was replaced. The FUE in groups SB, SBUE, and CLE was held in the third middle of the etching of the enamel surface using 37\% phosphoric acid for 30 seconds and removed with water for the same period. The FUWE groups SBUWE and CLWE did not receive previous treatment with phosphoric acid.

The adhesive systems were light cured for 20 seconds with the aid of Optilux 501 (Demetron ${ }^{\circledR}$ ) apparatus at a light intensity of $600 \mathrm{~mW} / \mathrm{cm}^{2}$. Plastic tubes (TYGON ${ }^{\circledR} 54-\mathrm{HL}$ Medical Tubing, Saint Gobain, Akron, OH, USA) with an internal diameter of $0.4 \mathrm{~mm}$ were placed on the enamel surface. The resin microhybrid composite TPH (Dentsply, Petrópolis, RJ, Brazil) was inserted into the tube and photopolymerization resin increment consisting of 40 seconds 
Table 1: General information about the tested adhesive systems

\begin{tabular}{|c|c|c|}
\hline Material & Composition & Form of application \\
\hline $\begin{array}{l}\text { Futurabond U } \\
\text { (VOCO, Cuxhaven, } \\
\text { Germany) }\end{array}$ & $\begin{array}{l}\text { Liquid 1: DMAs, fumed silica, acid-modified } \\
\text { methacrylates (MDP), camphorquinone, BHT, UDMA } \\
\text { Liquid 2: Ethanol, water, DC catalyst }\end{array}$ & $\begin{array}{l}1 \text { Mix the liquids contained in the blister. } \\
2 \text { Single application, active frictional manner with the } \\
\text { aid of microbrush for } 20 \text { seconds. } \\
3 \text { Light curing for } 20 \text { seconds. }\end{array}$ \\
\hline $\begin{array}{l}\text { Adper Single Bond } \\
\text { Universal (3M/ } \\
\text { ESPE, St. Paul, } \\
\text { MN, Estados } \\
\text { Unidos) }\end{array}$ & $\begin{array}{l}\text { Bis-GMA, HEMA, decamethylene DMA, ethanol, } \\
\text { water, silane-treated silica, 2-propenoic acid, } \\
\text {-methyl-, reaction products with 1,10-decanediol and } \\
\text { phosphorous oxide, copolymer of acrylic and itaconic } \\
\text { acid, dimethylaminobenzoate(-4), camphorquinone, } \\
\text { (dimethylamino)ethyl methacrylate, methyl ethyl ketone }\end{array}$ & $\begin{array}{l}1 \text { Single application, active frictional manner with the } \\
\text { aid of microbrush for } 15 \text { seconds. } \\
2 \text { Light curing for } 20 \text { seconds. }\end{array}$ \\
\hline $\begin{array}{l}\text { Adper Single Bond } \\
2 \text { (3M/ESPE, St. } \\
\text { Paul, MN, USA) }\end{array}$ & $\begin{array}{l}\text { Bis-GMA, HEMA, DMAs, ethanol, water, a new } \\
\text { photoinitiator system with functional polymer } \\
\text { methacrylate, and polyacrylic acids }\end{array}$ & $\begin{array}{l}1 \text { Acid conditioning for } 30 \text { seconds. } \\
2 \text { Acid removal with water for } 30 \text { seconds. } \\
3 \text { Enamel drying with compressed air for } 10 \text { seconds. } \\
4 \text { Application of the only actively frictional adhesive } \\
\text { with the aid of microbrush for } 15 \text { seconds. } \\
5 \text { Light curing for } 20 \text { seconds. }\end{array}$ \\
\hline $\begin{array}{l}\text { Clearfil (KURARAY, } \\
\text { Sakazu, Kurashiki, } \\
\text { Okayama, Japan) }\end{array}$ & $\begin{array}{l}\text { Primer: HEMA, MDP, hydrophilic DMA, water, } \\
\text { ethanol, dl-camphorquinone, N,N-diethanol-p-toluidine; } \\
\text { Adhesive: HEMA, MDP, Bis-GMA, hydrophilic DMA, } \\
\text { dl-camphorquinone, N,N-diethanol-p-toluidine, } \\
\text { silanized colloidal silica }\end{array}$ & $\begin{array}{l}1 \text { Application of frictional mode active primer with the } \\
\text { help of microbrush for } 20 \text { seconds. } \\
2 \text { Take air jet to the back. } \\
3 \text { Active bond application with the aid of microbrush } \\
\text { for } 20 \text { seconds. } \\
4 \text { Light curing for } 20 \text { seconds. }\end{array}$ \\
\hline
\end{tabular}

UDMA: Urethane dimethacrylate; Bis-GMA: Bisphenol-A diglycidyl ether dimethacrylate; DMA: Dimethacrylate; HEMA: Hydroxyethyl methacrylate

at a light intensity of $600 \mathrm{~mW} / \mathrm{cm}^{2}$ was held for a total of four composite restorations per sample. All samples were stored in distilled water at $37^{\circ} \mathrm{C}$ for 24 hours. After this period, the tubes were removed carefully with the aid of a no. 11 scalpel blade.

For microshear test, samples were placed in a metal base, and a stainless steel wire (Morelli Ortodontia, São Paulo, Brazil) with $0.2 \mathrm{~mm}$ diameter was placed around the resin cylinder, in line with the adhesive bonding interface, allowing the stay force vector parallel to the sample surface. The test was conducted in the universal testing machine EMIC (DL2000) with the speed of $0.5 \mathrm{~mm} /$ minute until its fracture. The average value of fracture of the four composite resin cylinders was used as the sample value of fracture.

The results were evaluated and statistically analyzed using Kruskal-Wallis and Mann-Whitney statistical tests followed by Bonferroni correction. After the bond strength tests, buccal surface through images evaluated in stereomicroscope ( $u$ Eye ${ }^{\circledR}$, Germany) was analyzed. The type of fracture that occurred in the rupture area between the enamel and the composite was determined according to the scores indicated in Table 2. The kappa

Table 2: Type of fracture after samples shear

\begin{tabular}{ll}
\hline Score & Fracture type \\
\hline 1 & Adhesive fracture-resin interface composite/adhesive \\
2 & Cohesive fracture in enamel \\
3 & Cohesive fracture in composite \\
4 & Mixed fracture-composite/enamel/adhesive \\
\hline
\end{tabular}

test was applied among the two evaluators and adequate reliability coefficient $(0.59)$ was considered.

\section{RESULTS}

Descriptive statistics of the different experimental groups is presented in Table 3, along with the minimum and maximum bond strength.

The Kruskal-Wallis statistical test showed significant statistical differences among the treated groups $(p<0.05)$. Mann-Whitney post hoc test identified that the groups that have been conditioned with acid obtained higher bond strength than the untreated group. It was also observed that the FUE group obtained the highest bond strength than other groups, while FUWE showed bond strength equivalent to that of other groups (Graph 1).

After the bond strength verification, there was a predominance of adhesive failures between the composite resin and adhesive (score 1). There was no enamel

Table 3: Mean (standard deviation, SD), maximum and minimum values of bond strength

\begin{tabular}{llllll}
\hline \multirow{2}{*}{$\begin{array}{l}\text { Experimental } \\
\text { groups }\end{array}$} & \multicolumn{5}{c}{ MPa } \\
\cline { 2 - 6 } & $n$ & Mean $(S D)$ & Max & Min & $I C$ \\
\hline FUE & 13 & $36.83(4.9)^{\mathrm{a}}$ & 43.6 & 28.0 & $33.81-39.85$ \\
FUWE & 13 & $28.43(1.9)^{\mathrm{b}}$ & 31.3 & 24.0 & $27.26-29.60$ \\
SB & 13 & $28.12(3.1)^{\mathrm{b}}$ & 35.0 & 24.0 & $26.22-30.02$ \\
SBUE & 13 & $30.33(3.3)^{\mathrm{b}}$ & 39.0 & 26.0 & $28.31-32.36$ \\
SBUWE & 13 & $18.40(2.2)^{\mathrm{c}}$ & 23.3 & 16.0 & $17.02-19.79$ \\
CLE & 13 & $27.85(4.7)^{\mathrm{b}}$ & 35.3 & 22.0 & $24.99-30.71$ \\
CLWE & 13 & $23.73(3.5)^{\mathrm{c}}$ & 28.5 & 19.0 & $21.57-25.88$ \\
\hline
\end{tabular}

*Different letters indicate statistically significant difference $(p<0.05)$ 
Influence of Different Etching Modes on Bond Strength to Enamel using Universal Adhesive Systems

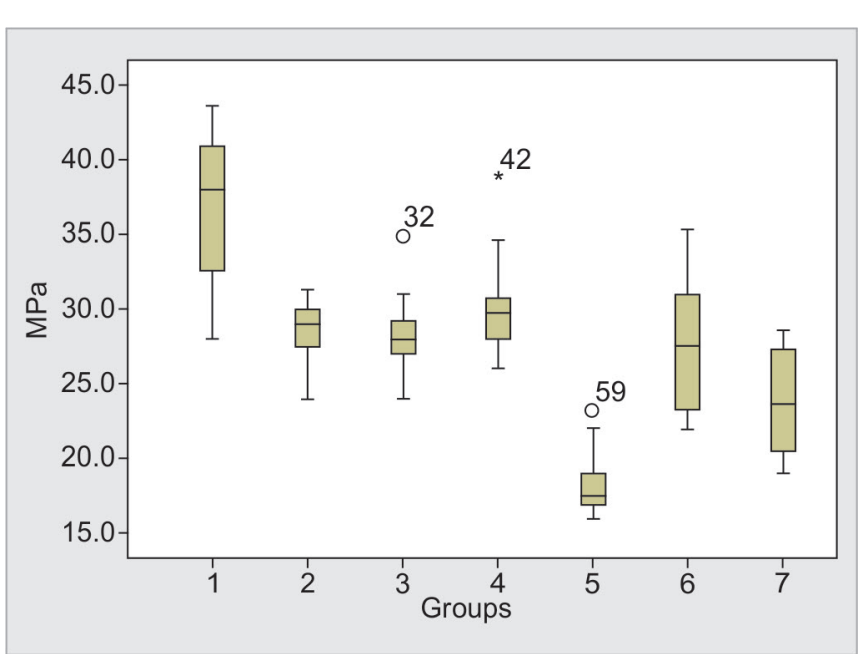

Graph 1: Mann-Whitney test mean, standard deviation, 25 percentile, and $75 \mathrm{MPa}$ of bond strength in different experimental groups

cohesive fracture (score 2) (Table 4). The reliability of the evaluation scores of the types of interrater fractures was assessed using the kappa coefficient (0.59), which was presented with an appropriate degree of evaluation of the agreement.

\section{DISCUSSION}

The use of total-etching adhesive systems has been effective and lasting for adhesion on dental enamel. ${ }^{7}$ However, with the development and incorporation of new materials and restorative techniques, it is necessary to carry out studies in order to validate the application of these materials in practice. In this study, it was observed that the total etching promoted an increase in bond strength before the use of evaluated adhesive systems. The universal adhesive system (SBU) had a behavior similar to self-etching (CL), and the universal multipurpose adhesive FU showed high bond strength values. Thus, the null hypothesis was rejected.

Bovine teeth were used because it is known that they are a viable alternative to carry out studies in vitro. ${ }^{12,13}$ Due to the difficulty in obtaining human teeth, this substitution has been viable as they present micromorphological characteristics similar to human teeth. ${ }^{14}$ The microshear test was performed in this study as it is a suitable means for measuring the bond strength of adhesive restorations, allows better analysis, and promotes a better stress distribution on the surface of samples. ${ }^{15}$

To study the bond strength of different adhesive systems, the adhesive system was applied actively and vigorously, ${ }^{10}$ since better adhesion results are obtained immediately or long-term.

The bond strength results found for the conventional adhesive system, the total-etch SB $(28.12 \pm 3.1 \mathrm{MPa})$, were almost similar to those already found in the literature. ${ }^{16}$
Table 4: Distribution of the scores by the type of fracture

\begin{tabular}{lllll}
\hline $\begin{array}{l}\text { Experimental } \\
\text { groups }\end{array}$ & \multicolumn{4}{c}{ Type of fracture - Scores (\%) } \\
\cline { 2 - 5 } & $1^{*}$ & $2^{* *}$ & $3^{* * *}$ & $4^{* * * *}$ \\
\hline G1 (FUE) & $13(100)$ & 0 & 0 & 0 \\
G2 (FUWE) & $11(85)$ & 0 & 0 & $2(15)$ \\
G3 (SB) & $13(100)$ & 0 & $0(0)$ & $0(0)$ \\
G4 (SBUE) & $10(77)$ & 0 & $1(8)$ & $2(15)$ \\
G5 (SBUWE) & $2(15)$ & 0 & $9(70)$ & $2(15)$ \\
G6 (CLE) & $2(15)$ & 0 & $4(31)$ & $7(54)$ \\
G7 (CLWE) & $11(85)$ & 0 & 0 & $2(15)$ \\
\hline
\end{tabular}

${ }^{*}$ Adhesive fracture - resin interface composite/adhesive; ${ }^{* *}$ Cohesive fracture in enamel; ${ }^{* * *}$ Cohesive fracture in composite; ${ }^{* * * *}$ Mixed fracture - composite/enamel/adhesive

The self-etch adhesive and multipurpose systems presented greater values of bond strength when enamel acid etching was performed, corroborating the literature and the manufacturer's recommendations. ${ }^{7,17}$ The pretreatment of the enamel with phosphoric acid increases the bond strength ${ }^{9,18}$ and results in the formation of longer resin tags and greater depth of penetration of the intact enamel adhesive. ${ }^{18}$ However, the depth of penetration of the adhesive does not necessarily predict increased bond strength. ${ }^{11}$

According to Borges et al, ${ }^{19}$ self-etch adhesive systems associated with the prior acid etching of the CLE enamel $(27.85 \pm 4.7)$ bond strength values are similar to the group treated with the conventional total-etch system. Self-etch systems are composed of weaker acidic monomers when compared with phosphoric acid; thus, the interprismatic enamel decalcifies up selectively, not promoting an improvement in the bond strength. ${ }^{17}$ The self-etch Clearfil SE adhesive has in its composition 10-metacriloiloxidecil dihydrogen phosphate (10-MDP), which is a monomer of functional phosphate ester. The phosphate group has the potential to interact with the hydroxyapatite, and it is able to form strong ionic bonds with calcium due to relatively low calcium dissolution rate that contributes significantly to the durability of the restorations. ${ }^{20,21}$

The evolution of self-etch systems made possible the development of universal adhesive systems, which in addition to other components also has the 10-MDP molecule. Thus, the coupling mechanism occurs due to a micromechanical and chemical nanointeraction. Nanolayers of 10-MDP molecules form a stable MDP-Ca salt, capable of making the adhesive interface more resistant to biodegradation. This factor may explain the documented clinical longevity of dentin interfaces, obtained from the use of adhesive systems based on 10-MDP. 22

Although studies show that acid etching improves the penetration of universal adhesives, it is worth remembering that in dentin, ${ }^{23}$ total etching systems remove 
the filler content exposing the organic matrix. Thus, it becomes more difficult to form the MDP-Ca salt, which ensures the chemical interaction of the nanomaterial. This chemical interaction, in the dentin, is favored by its partial demineralization, preserving the integrity of collagen fibers that interact chemically with the 10-MDP forming a stable layer. Moreover, the lowest rate of dissolution of calcium salts that carries in high potential of chemical bond also favors the interaction. ${ }^{1,22}$

Among the universal adhesive systems studied, FUE was the one with the highest bond strength values $(36.83 \pm 4.9 \mathrm{MPa})$, and the FUWE group $(28.43 \pm 1.9 \mathrm{MPa})$ showed similar results to the adhesive full-etch system. Therefore, the behavior of multipurpose adhesive systems seems to be material dependent, ${ }^{24}$ since SBUE group presented bond strength values lower than FU group respectively, the SBUWE $(18.40 \pm 2.2)$ and conditioning group $(30.33 \pm 3.3)$.

In addition, butylated hydroxytoluene (BHT), a component of FU, acts as an inhibitor, preventing spontaneous polymerization system and promoting increased durability of adhesion to resins, which could explain the higher values of resistance of this adhesive system. ${ }^{25}$

Therefore, it was observed that among the adhesive systems evaluated, the multipurpose systems have their bond strength varying according to the composition of the material and that even if the enamel etching is necessary to obtain better retention standards, only FU system showed behavior similar to the control group. Future studies are needed to verify the performance of this new category of long-term materials and their clinical effectiveness. It was found that the most prevalent type of fracture occurred in the interface composite resin/ adhesive (score 1) in the study group.

According to the data and statistical analysis applied to the results, it is concluded that the enamel acid etching provides the best bond strength results for the self-etch and multipurpose systems. Also, it is possible to highlight that the multipurpose systems have varied their bond strength according to the brand and composition of the material.

\section{ACKNOWLEDGMENT}

The authors are grateful for the support provided by the Foundation of Research and Scientific and Technological Development of Maranhão (FAPEMA) for the publication of this manuscript and the Institutional Scholarship Program for Scientific Initiation PIBIC-CEUMA for preparation of this study.

\section{REFERENCES}

1. Van Meerbeek B, Yoshihara K, Yoshida Y, Mine A, De Munck J, Van Landuyt KL. State of the art of self-etch adhesives. Dent Mater 2011 Jan;27(1):17-28.
2. JuniorMM,RochaEP,Anchieta RB,ArchangeloCM, LuersenMA. Etch and rinse versus self-etching adhesives systems: Tridimensional micromechanical analysis of dentin/adhesive interface. Int J Adhes Adhesives 2012 Jun;35:114-119.

3. Hiraishi N, Breschi L, Prati C, Ferrari M, Tagami J, King NM. Technique sensitivity associated with air-drying of HEMAfree, single-bottle, one-step self-etch adhesives. Dent Mater J 2007 Apr;23(4):498-505.

4. Ozer F, Blatz MB. Self-etch and etch-and-rinse adhesive systems in clinical dentistry. Compend Contin Educ Dent 2013 Jan;34(1):12-14.

5. Perdigão J, Reis A, Loguercio AD. Dentin adhesion and MMPs: a comprehensive review. J Esthet Restor Dent 2013 Aug;25(4):219-241.

6. Jacques P, Hebling J. Effect of dentin conditioners on the microtensile bond strength of a conventional and a selfetching primer adhesive system. Dent Mater 2005 Feb;21(2): 103-109.

7. Sabatini C. Effect of phosphoric acid etching on the shear bond strength of two self-etch adhesives. J Appl Oral Sci 2013 Jan-Feb;21(1):56-62.

8. Muñoz MA, Luque I, Hass V, Reis A, Loguercio AD, Bombarda NH. Immediate bonding properties of universal adhesives to dentine. J Dent 2013 May;41(5):404-411.

9. McLean, Meyers E, Guillory V, Vandewalle K. Enamel bond strength of new universal adhesive bonding agents. Oper Dent 2015 Jul-Aug;40(4):410-417.

10. Perdigão J, Loguercio AD. Universal or multi-mode adhesives: Why and how? Adhes Dent 2014 Apr;16(2):193-194.

11. Heintze SD, Zimmerli B. Relevance of in vitro tests of adhesive and composite dental materials. A review in 3 parts. Part 3: In vitro tests of adhesive systems. Schweiz Monatsschr Zahnmed 2011;121(11):1024-1040.

12. Schilke R, Lisson JA, Bauk O, Geurtsen W. Comparison of the number and diameter of dentinal tubules in human and bovine dentine by scanning electron microscopic investigation. Arch Oral Biol 2000 May;45(5):355-361.

13. Krifka S, Börzsönyi A, Koch A, Hiller KA, Schmalz G, Friedl $\mathrm{KH}$. Bond strength of adhesive system to dentin and enamel - Human vs. bovine primary teeth in vitro. Dent Mater J 2008 Jul;24(7):888-894.

14. Poggio C, Scribante A, Della Zoppa F, Colombo M, Beltrami R, Chiesa M. Shear bond strength of one-step self-etch adhesives to enamel: effect of acid pretreatment. Dent Traumatol 2014 Feb;30(1):43-48.

15. Nakamichi M, Iwaku M, Fusayama T. Bovine teeth as a possible substitute in the adhesion test. J Dent Res 1983 Oct;62(10):1076-1081.

16. Beloica M,GoracciC,CarvalhoCA, RadovicI,Margvelashvili M, Vulicevic ZR, Ferrari M. Microtensile vs microshear bond strength of all-in-one adhesives to unground enamel. J Adhes Dent 2010 Dec;12(6):427-433.

17. Goswami M, Singh A. Comparative evaluation of shear bond strength of composite resin bonded to acid etched or Nd:Yag lased enamel. J Indian Soc Pedod Prev Dent 2011 Apr-Jun;29(2):140-143.

18. Devarasa GM, Subba Reddy VV, Chaitra NL. Self etching adhesive on intact enamel. J Indian Soc Pedod Prev Dent 2012 Apr-Jun;30(2):133-138.

19. Borges MAP, Matos IC, Dias KRHC. Influence of two selfetching primer systems on enamel adhesion. Braz Dent J 2007;18(2):113-118. 
20. Fukegawa D, Hayakawa S, Yoshida Y, Suzuki K, Osaka A, Van Meerbeek B. Chemical interaction of phosphoric acid ester with hydroxyapatite. J Dent Res 2006 Oct;85(10): 941-944.

21. Fujita K, Ma S, Aida M, Maeda T, Ikemi T, Hirata M, Nishiyama N. Effect of reacted acidic monomer with calcium on bonding performance. J Dent Res 2011 May;90(5): 607-612.

22. Yoshida Y, Yoshihara K, Nagaoka N, Hayakawa S, Torii Y, Ogawa T, Osaka A, Meerbeek BV. Self-assembled nano-layering at the adhesive interface. J Dent Res 2012 Apr;91(4):376-381.
23. Wagner A, Wendler M, Petschelt A, Belli R, Lohbauer U. Bonding performance of universal adhesives in different etching modes. J Dent 2014 Jul;42(7):800-807.

24. Muñoz M, Luque-Martinez I, Malaquias P, Hass V, Reis A, Campanha N, Loguercio A. In vitro longevity of bonding properties of universal adhesives to dentin. Oper Dent 2015 May-Jun;40(3):282-292.

25. Wang Z, Landis FA, Giuseppetti AA, Lin-Gibson S, Chiang MY. Simultaneous measurement of polymerization stress and curing kinetics for photo-polymerized composites with high filler contents. Dent Mater 2014 Dec;30(12): 1316-1324. 